\title{
PROSTATE CANCER
}

\section{Timing is everything for docetaxel therapy}

T

results of two phase III trials recently published in The Lancet

Oncology provide new information

on when to administer docetaxel to

patients with prostate cancer.

Traditionally given to men with castration-resistant prostate cancer (CRPC), the GETUG-AFU 15 trial was designed to investigate whether docetaxel could improve outcomes if administered earlier in the disease course-specifically, alongside androgen deprivation therapy

(ADT) for patients with newly metastatic, hormone-sensitive disease. The results were not supportive of a role for docetaxel in this setting.

For this trial, 385 men with radiologically proven metastatic prostate cancer were recruited from 29 centres in France and one in Belgium and randomized to receive ADT alone (orchiectomy or luteinising hormone-releasing hormone agonists, alone or combined with nonsteroidal antiandrogens) or in combination with docetaxel $\left(75 \mathrm{mg} / \mathrm{m}^{2}\right.$ on the first day of a 3-week cycle). The primary end point, overall survival, was not significantly different between groups, at 58.9 months for patients who received ADT plus docetaxel compared with 54.2 months for those who received ADT alone (HR 1.01, 95\% CI 0.75-1.36; $P=0.955$ ).

On the other hand, addition of docetaxel to ADT did significantly improve biochemical progression-free survival (a secondary end point), which was 22.9 months for the combined treatment group and 12.9 months for those who received ADT alone (HR 0.72, 95\% CI 0.57-0.91; $P=0.005)$. Although the investigators state that the known correlation between PSA progression and overall survival make this a clinically relevant finding, they also note the controversial use of PSA as a surrogate end point.

Particularly discouraging are the data concerning toxicities. Not only did 21\% of men who received docetaxel plus ADT discontinue treatment owing to toxic effects, but a total of 72 serious events (assessed with the Common Toxicity Criteria of the National Cancer Institute version 3) were reported in this patient group; no serious events were reported by men who received ADT alone. The most frequently reported serious event was neutropenia, reported at grade $3-4$ by almost a third of men receiving docetaxel. Furthermore, four deaths (5\%) in the ADT plus docetaxel group were attributed to treatment-related toxic effects compared with none in the group given ADT alone.

Overall, these data indicate that docetaxel cannot be recommended in this setting, although other studies might help clarify the true survival benefit of docetaxel plus ADT for men with hormone-sensitive prostate cancer. The investigators of GETUG-AFU 15 list sample size as a limitation of the trial and suggest it might have been underpowered to detect a small difference in overall survival. The Eastern Cooperative Oncology Group E3805 trial and the Medical Research Council's STAMPEDE trial (Systemic Therapy in Advanced or Metastatic Prostate Cancer: Evaluation of Drug Efficacy) are currently underway with estimated patient enrolment numbers of 780 and 4,000, respectively, the results of which will hopefully contribute to the discussion.

As Christopher Sweeney from the Dana-Farber Cancer Institute says in an accompanying commentary, this study "marks the dawn of a new era." Multiple agents have been shown to extend survival of patients with CRPC and now they are being tested in patients with newly metastatic disease; similar studies are currently underway for abiraterone, sipuleucel-T, enzalutamide, and radium223 chloride. Time will tell whether these drugs provide better outcomes than docetaxel in this setting.

The second trial published in The Lancet Oncology reports a 2-weekly docetaxel schedule for patients with CRPC as an alternative to the commonly used 3-weekly regimen.

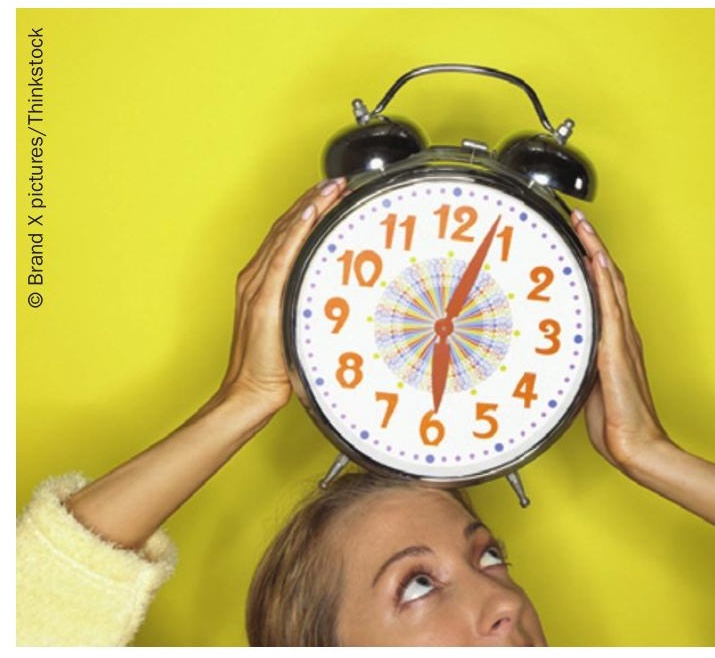

Despite becoming the standard first-line treatment of CRPC, 3-weekly intravenous injections of docetaxel are associated with a number of adverse effects including neutropenic fever, fatigue, diarrhoea, sensory neuropathy and alopecia. In an attempt to find a dosing schedule that might be better tolerated, a research group from Finland randomized patients with CRPC to receive either $75 \mathrm{mg} / \mathrm{m}^{2}$ on the first day of a 3 -week cycle $(n=184)$ or a lower dose of $50 \mathrm{mg} / \mathrm{m}^{2}$ on the first day of a 2 -week cycle $(n=177)$.

The 2-weekly regimen was associated with a longer time to treatment failure than the 3-weekly regimen (5.6 months versus 4.9 months; $P=0.014)$, and a lower rate of grade $3-4$ adverse events (53\% versus $35 \%$ for neutropenia). Treatment safety is an important consideration for patients with CRPC and these findings suggest that a 2-weekly regimen of docetaxel is feasible for men with comorbidities who are unlikely to tolerate large single doses of docetaxel.

Sarah Payton

Original articles Gravis, G. et al. Androgen-deprivation
therapy alone or with docetaxel in non-castrate metastatic
prostate cancer (GETUG-AFU 15): a randomised, open-
label, phase 3 trial. Lancet Oncol. doi:10.1016/S1470-
2045(12)70560-0 | Kellokumpu-Lehtinen, P. L. et al.
2-weekly versus 3-weekly docetaxel to treat castration-
resistant advanced prostate cancer: a randomised,
phase 3 trial. Lancet Oncol. doi:10.1016/S1470-
2045(12)70537-5

